\title{
Positive solutions of second-order linear difference equation with variable delays
}

Hajnalka Peics*

\section{${ }^{*}$ Correspondence:}

peics@gf.uns.ac.rs

Faculty of Civil Engineering,

University of Novi Sad, Subotica,

Serbia

\begin{abstract}
In this paper we consider the second-order linear difference equations with variable delays

$$
\Delta^{2} a(n)+\sum_{i=1}^{m} P_{i}(n) a\left(n-k_{i}(n)\right)=0, \quad n \geq n_{0}
$$

where $n_{0}, n \in \mathbf{N}, \mathbf{N}$ is the set of positive integers. Using the method of Riccati transform and the generalized characteristic equations, we give sufficient conditions for the existence of positive solutions.

MSC: Primary 39A11; secondary 39A12
\end{abstract}

Keywords: second-order linear difference equation; variable delays; existence; positive solutions; non-oscillatory solutions; sufficient conditions

\section{Introduction}

In the past few years, oscillation and non-oscillation theory of second-order linear and nonlinear difference equations has attracted considerable attention, and we refer the reader to the papers of Diblik et al. [1], El-Sheik et al. [2], He [3], Huiqin and Zhen [4], Koplatadze and Kvinikadze [5], Krasznai et al. [6], Li et al. [7], Liu and Cheng [8], Medina and Pituk [9], Tang [10], Tang and Yu [11], Zhang [12], Zhang and Li [13], and the references therein. For comprehensive treatment, see the papers by Bastinec et al. [14-17], Čermak [18, 19], Deng [20], Došly and Fišnarová [21], Hille [22], Jaroš and Stavroulakis [23], Thandapani et al. [24], Yang [25] and the monographs [26, 27]. Some sharp conditions of Hille-type criterion on the existence of oscillatory and non-oscillatory solutions of second-order differential equations are given by Kusano et al. [28, 29], by Péics and Karsai [30], by Bastinec et al. [31] and by Berezansky et al. [32]. Some sufficient conditions of oscillation and non-oscillation of second-order difference equations can be found in the papers by Lei [33], by Li and Jiang [34], by Zhou and Zhang [35], and the references therein.

Consider the second-order delay differential equation of the form

$$
x^{\prime \prime}(t)+\sum_{i=1}^{m} p_{i}(t) x\left(t-\tau_{i}(t)\right)=0
$$

for $t_{0} \leq t<T \leq \infty$, where the following hypotheses are satisfied:

\section{Springer}

2013 Peics; licensee Springer. This is an Open Access article distributed under the terms of the Creative Commons Attribution License (http://creativecommons.org/licenses/by/2.0), which permits unrestricted use, distribution, and reproduction in any medium, provided the original work is properly cited. 
$\left(\mathrm{H}_{1}^{*}\right) p_{i} \in C\left[\left[t_{0}, T\right), \mathbf{R}\right], i=1,2, \ldots, m$;

$\left(\mathrm{H}_{2}^{*}\right) \tau_{i} \in C\left[\left[t_{0}, T\right), \mathbf{R}^{+}\right], i=1,2, \ldots, m$.

As a special case we can formulate the following results given in [30] for half-linear delay differential equations.

Theorem A (see Theorem 2 in [30]) Assume that $\left(\mathrm{H}_{1}^{*}\right)$ and $\left(\mathrm{H}_{2}^{*}\right)$ hold and there exists a positive function $\mu(t)$ for $t \geq t_{0}$ such that

$$
\int_{t}^{\infty}\left[\mu(t)^{2}+\sum_{i=1}^{m}\left|p_{i}(s)\right| \exp \left(\int_{s-\tau_{i}(s)}^{s} \mu(\xi) d \xi\right)\right] d s \leq \mu(t)
$$

holds for t large enough. Then equation (1) has a positive solution.

Theorem B (see Corollary 2 in [30]) Assume that $\left(\mathrm{H}_{1}^{*}\right)$ and $\left(\mathrm{H}_{2}^{*}\right)$ hold and the functions $\tau_{i}(t)$, where $i=1,2, \ldots, m$, are bounded. If

$$
\limsup _{t \rightarrow \infty} t \int_{t}^{\infty} \sum_{i=1}^{m}\left|p_{i}(s)\right| d s \leq \frac{1}{4}
$$

then equation (1) has a positive solution.

Consider the second-order difference equation of the form

$$
\Delta^{2} a(n)+P(n) a(n)=0, \quad n \geq n_{0},
$$

where $n_{0} \in \mathbf{N}, \mathbf{N}$ is the set of positive integers and $\{P(n)\}$ is a sequence of real numbers.

The following result is a sufficient condition for the existence of non-oscillatory solutions of equation (2) and it is demonstrated in the paper [35].

Theorem C (see Lemma 4 in [35]) Assume that $\{P(n)\}$ is a real sequence with $P(n) \geq 0$ for all $n \geq n_{0}$. If

$$
n \sum_{i=n+1}^{\infty} P_{i}(s) \leq \frac{1}{4}
$$

for all large $n$, then equation (2) has a non-oscillatory solution.

Consider now the second-order difference equation with variable delays

$$
\Delta^{2} a(n)+\sum_{i=1}^{m} P_{i}(n) a\left(n-k_{i}(n)\right)=0, \quad n \geq n_{0}
$$

where $n_{0} \in \mathbf{N}, \mathbf{N}$ is the set of positive integers and the following hypotheses are satisfied:

$\left(\mathrm{H}_{1}\right) \quad\left\{P_{i}(n)\right\}$ is a sequence of real numbers for $i=1,2, \ldots, m$ and $n \geq n_{0}$;

$\left(\mathrm{H}_{2}\right) \quad\left\{k_{i}(n)\right\}$ is a sequence of positive integers such that $k_{i}(n) \leq n$ for $i=1,2, \ldots, m$ and $n \geq n_{0}$. 
Let $M \geq n_{0}$ be an arbitrary natural number and set

$$
\mathbf{N}_{M}=\{n \mid n \in \mathbf{N}, n \geq M\} .
$$

By a solution of equation (3) we mean a sequence of real numbers $\{a(n)\}$ defined for $n \in$ $\mathbf{N}_{M}$, which satisfies equation (3) for all $n \in \mathbf{N}_{M}$. A nontrivial solution $\{a(n)\}$ of equation (3) is said to be oscillatory if for every $v>n_{0}$, there exists an $n \geq v$ such that $a(n) a(n+1) \leq 0$. Otherwise, it is non-oscillatory. Thus, a non-oscillatory solution is either eventually positive or eventually negative.

The basic idea in the paper is to use the Riccati transformation technique by the substitution

$$
\lambda(n)=\frac{\Delta a(n)}{a(n)},
$$

where $\{a(n)\}$ is a solution sequence of equation (3). This transformation leads to the generalization of the Riccati-type equation associated with equation (3). The aim of this paper is to prove theorems for the existence of positive solutions of equation (3), using the Riccatitype equation associated with equation (3). The obtained results are discrete analogues of results given for some differential equations and generalize results given for second-order linear difference equations with constant delays or without delays.

Let $\mathbf{R}^{\infty}:=\left\{\{\xi(n)\}_{n=1}^{\infty}: \xi(n) \in \mathbf{R}, n=1,2, \ldots\right\}$ and

$$
\ell^{\infty}:=\left\{\{\xi(n)\} \in \mathbf{R}^{\infty}:|\xi(n)| \leq \mu(n), n \in \mathbf{N}_{M}\right\},
$$

where $\{\mu(n)\}$ is a fixed positive bounded sequence.

Let $\left\{x_{p}(n)\right\}$ denote the sequence of sequences defined for all natural numbers $n \in\left[n_{0}, \infty\right)$ and $p=1,2,3, \ldots$.

Theorem $\mathbf{D}$ (Schauder-Tychonoff, see [36-38]) Let $F=\ell^{\infty}$, and let $\{\mu(n)\}$ be a fixed positive bounded sequence. Let $S$ be a mapping of $F$ into itself with the properties:

(i) $S$ is continuous in the sense that if $x_{p}(n) \in F$ for all natural number $n \geq n_{0}$, $p=1,2, \ldots$, and $x_{p}(n) \rightarrow x(n), p \rightarrow \infty$, uniformly on every compact subinterval of $\left[n_{0}, \infty\right)$, then $S x_{p}(n) \rightarrow S x(n), p \rightarrow \infty$, uniformly on every compact subinterval of $\left[n_{0}, \infty\right)$;

(ii) the sequences in the image set SF are bounded at every point of $\left[n_{0}, \infty\right)$.

Then the mapping $S$ has at least one fixed point in $F$.

\section{Main results}

First, we apply the Riccati transformation and show the relationship between equation (3) and the Riccati-type equation (4).

Lemma 1 Assume that conditions $\left(\mathrm{H}_{1}\right)$ and $\left(\mathrm{H}_{2}\right)$ hold. Then the following statements are equivalent.

(a) Equation (3) has an eventually positive solution. 
(b) There is a sequence $\{\lambda(n)\}, n \in \mathbf{N}_{M}$, for some $M \geq n_{0}$ such that $\lambda(n)+1>0$ for all $n \in \mathbf{N}_{M}$ and $\{\lambda(n)\}$ satisfies the Riccati-type equation

$$
\Delta \lambda(n)+\lambda(n+1) \lambda(n)+\sum_{i=1}^{m} P_{i}(n) \prod_{j=n-k_{i}(n)}^{n-1} \frac{1}{\lambda(j)+1}=0 \quad \text { for } n \in \mathbf{N}_{M}
$$

Proof (a) $\Rightarrow$ (b): Let $\{a(n)\}$ be the solution of difference equation (3) and suppose, according to the hypotheses, that $a(n)>0$ for $n \geq M \geq n_{0}$. It will be shown that the sequence $\{\lambda(n)\}$ defined by

$$
\lambda(n)=\frac{\Delta a(n)}{a(n)}, \quad n \in \mathbf{N}_{M},
$$

is a solution of the Riccati-type equation (4) for $n \in \mathbf{N}_{M}$. Expressing $\Delta a(n)$ from (5) and applying the difference operator to the transformed equality, we get that

$$
\Delta a(n)=a(n) \lambda(n) \quad \text { and } \quad \Delta[\Delta a(n)]=a(n) \lambda(n+1) \lambda(n)+a(n) \Delta \lambda(n) .
$$

It follows from the first equality of (6) that

$$
a(n+1)=a(n)(\lambda(n)+1) \quad \text { and } \quad a(n)=a(M) \prod_{j=M}^{n-1}(\lambda(j)+1) \quad \text { for } n \in \mathbf{N}_{M}
$$

and hence the following equalities are valid:

$$
\frac{a\left(n-k_{i}(n)\right)}{a(n)}=\frac{a(M) \prod_{j=M}^{n-k_{i}(n)-1}(\lambda(j)+1)}{a(M) \prod_{j=M}^{n-1}(\lambda(j)+1)}=\prod_{j=n-k_{i}(n)}^{n-1} \frac{1}{\lambda(j)+1} .
$$

By dividing both sides of difference equation (3) by $a(n)$, we obtain that

$$
\frac{\Delta[\Delta a(n)]}{a(n)}+\sum_{i=1}^{m} P_{i}(n) \frac{a\left(n-k_{i}(n)\right)}{a(n)}=0
$$

In virtue of the equalities (6) and (7), we obtain that

$$
\frac{a(n) \lambda(n+1) \lambda(n)+a(n) \Delta \lambda(n)}{a(n)}+\sum_{i=1}^{m} P_{i}(n) \prod_{j=n-k_{i}(n)}^{n-1} \frac{1}{\lambda(j)+1}=0
$$

After reducing the first term, we get that

$$
\lambda(n+1) \lambda(n)+\Delta \lambda(n)+\sum_{i=1}^{m} P_{i}(n) \prod_{j=n-k_{i}(n)}^{n-1} \frac{1}{\lambda(j)+1}=0
$$

and we conclude that the sequence $\{\lambda(n)\}$ satisfies the Riccati-type equation (4), and the first part of the proof is complete. 
(b) $\Rightarrow$ (a): Let now $\{\lambda(n)\}$ be a solution sequence of Riccati-type equation (4) for $n \in \mathbf{N}_{M}$ such that $\lambda(n)+1>0$ for all $n \in \mathbf{N}_{M}$. We show by direct substitution that the sequence defined by

$$
a(n)=\prod_{j=M}^{n-1}(\lambda(j)+1) \quad \text { for } n \in \mathbf{N}_{M}
$$

is the positive solution of difference equation (3). Completing the following transformations, we get

$$
\begin{aligned}
\Delta[\Delta a(n)] & =a(n)(\lambda(n+1) \lambda(n)+\Delta \lambda(n)) \\
& =a(n)\left(-\sum_{i=1}^{m} P_{i}(n) \prod_{j=n-k_{i}(n)}^{n-1} \frac{1}{\lambda(j)+1}\right) \\
& =-a(n) \sum_{i=1}^{m} P_{i}(n) \frac{a\left(n-k_{i}(n)\right)}{a(n)} \\
& =-\sum_{i=1}^{m} P_{i}(n) a\left(n-k_{i}(n)\right)
\end{aligned}
$$

for $n \in \mathbf{N}_{M}$ and the proof of Lemma 1 is complete.

Now, we introduce an antidifference equation associated with the Riccati-type equation (4), and we show in the main results that it is very useful to study the antidifference equation instead of equation (4).

Lemma 2 Assume that conditions $\left(\mathrm{H}_{1}\right)$ and $\left(\mathrm{H}_{2}\right)$ hold. Then the following statements are equivalent.

(a) There is a solution sequence $\{\lambda(n)\}, n \in \mathbf{N}_{M}$, of the Riccati-type equation (4) for some $M \geq n_{0}$ such that $\lambda(n)+1>0$ for all $n \in \mathbf{N}_{M}$ and

$$
\sum_{\ell=n}^{\infty}\left(\sum_{i=1}^{m} P_{i}(\ell) \prod_{j=\ell-k_{i}(\ell)}^{\ell-1} \frac{1}{\lambda(j)+1}\right)<\infty
$$

(b) There is a sequence $\{\omega(n)\}, n \in \mathbf{N}_{M}$, for some $M \geq n_{0}$ such that $\omega(n)+1>0$ for all $n \in \mathbf{N}_{M}$ and

$$
\omega(n)=\sum_{\ell=n}^{\infty} \omega(\ell+1) \omega(\ell)+\sum_{\ell=n}^{\infty}\left(\sum_{i=1}^{m} P_{i}(\ell) \prod_{j=\ell-k_{i}(\ell)}^{\ell-1} \frac{1}{\omega(j)+1}\right)
$$

Proof (a) $\Rightarrow$ (b): Let the sequence $\{\lambda(n)\}$, defined by formula $\lambda(n)=\omega(n)$ for $n \in \mathbf{N}_{M}$, be a solution of the Riccati-type equation (4) for $n \in \mathbf{N}_{M}$ with the property (9). Let $n \geq M$ be a natural number fixed arbitrarily, and let us sum up both sides of the Riccati-type equation (4) from $n$ to $M_{1}-1$, where $M_{1}>n$. Then we get the equality

$$
\omega\left(M_{1}\right)-\omega(n)+\sum_{\ell=n}^{M_{1}-1} \omega(\ell+1) \omega(\ell)+\sum_{\ell=n}^{M_{1}-1}\left(\sum_{i=1}^{m} P_{i}(\ell) \prod_{j=\ell-k_{i}(\ell)}^{\ell-1} \frac{1}{\omega(j)+1}\right)=0 .
$$


We claim that

$$
\sum_{\ell=n}^{\infty} \omega(\ell+1) \omega(\ell)<\infty
$$

To this end, assume the contrary statement that $\sum_{\ell=n}^{\infty} \omega(\ell+1) \omega(\ell)=\infty$. Now, in view of (11) and the statement $\sum_{\ell=n}^{\infty} \omega(\ell+1) \omega(\ell)=\infty$, there are natural numbers $M_{1}$ and $M^{*}$ large enough such that $M_{1}>M^{*}$ and

$$
\begin{aligned}
& \omega\left(M_{1}\right)+\sum_{\ell=M^{*}}^{M_{1}-1} \omega(\ell+1) \omega(\ell) \\
& =\omega(n)-\sum_{\ell=n}^{M^{*}-1} \omega(\ell+1) \omega(\ell)-\sum_{\ell=n}^{M_{1}-1}\left(\sum_{i=1}^{m} P_{i}(\ell) \prod_{j=\ell-k_{i}(\ell)}^{\ell-1} \frac{1}{\omega(j)+1}\right) \leq-1
\end{aligned}
$$

for $M_{1}>M^{*} \geq n$, or, equivalently,

$$
-\omega\left(M_{1}\right) \geq \sum_{\ell=M^{*}}^{M_{1}-1} \omega(\ell+1) \omega(\ell)+1 .
$$

Because of the substitution (5), it follows that

$$
-\frac{a\left(M_{1}+1\right)}{a\left(M_{1}\right)}=-\omega\left(M_{1}\right)-1 \geq \sum_{\ell=M^{*}}^{M_{1}-1} \omega(\ell+1) \omega(\ell) .
$$

Since $M_{1}>M$ is an arbitrary large number, then the inequality

$$
\sum_{\ell=M^{*}}^{M_{1}-1} \omega(\ell+1) \omega(\ell)<0
$$

holds. If $M_{1} \rightarrow \infty$, then the inequality $\sum_{\ell=M^{*}}^{\infty} \omega(\ell+1) \omega(\ell)<0$ must hold, which contradicts the assumption

$$
\sum_{\ell=n}^{\infty} \omega(\ell+1) \omega(\ell)=\infty
$$

We now let $M_{1} \rightarrow \infty$ in (11). Using inequalities (12) and (9), we find that $\omega\left(M_{1}\right)$ tends to a finite limit $\omega_{\infty}$. But $\omega_{\infty}$ must be zero because in the other case inequality (12) would fail to hold. These argumentations complete the first part of the proof.

(b) $\Rightarrow$ (a): Assume that there is a sequence $\{\omega(n)\}$ which satisfies equation (10) for $n \in$ $\mathbf{N}_{M}$, where $M \geq n_{0}$ is an arbitrary natural number such that $\omega(n)+1>0$ for all $n \in \mathbf{N}_{M}$. Applying the difference operator to both sides of equation (10), we show that the sequence $\{\lambda(n)\}$, defined by formula $\lambda(n)=\omega(n)$ for $n \in \mathbf{N}_{M}$, is a solution of the Riccati-type equation (4) for $n \in \mathbf{N}_{M}$, and it satisfies assumption (9). The proof of the theorem is complete.

Now we can formulate the main theorem.

Theorem 1 Assume that conditions $\left(\mathrm{H}_{1}\right)$ and $\left(\mathrm{H}_{2}\right)$ hold. Then the following statements are equivalent. 
(a) There exists a natural number $M \geq n_{0}$ and there exist the real sequences $\{\beta(n)\}$ and $\{\gamma(n)\}$ such that $-1<\beta(n) \leq \gamma(n), \sup _{n} \gamma(n)<\infty$ for $n \in \mathbf{N}_{M}$,

$$
\sum_{\ell=n}^{\infty}\left(\sum_{i=1}^{m}\left|P_{i}(\ell)\right| \prod_{j=\ell-k_{i}(\ell)}^{\ell-1} \frac{1}{\beta(j)+1}\right)<\infty
$$

and such that

$$
\beta(n) \leq \xi(n) \leq \gamma(n) \quad \text { implies that } \quad \beta(n) \leq S \xi(n) \leq \gamma(n)
$$

for $n \in \mathbf{N}_{M}$ and for real sequences $\{\xi(n)\}$, where

$$
S \xi(n)=\sum_{\ell=n}^{\infty} \xi(\ell+1) \xi(\ell)+\sum_{\ell=n}^{\infty}\left(\sum_{i=1}^{m} P_{i}(\ell) \prod_{j=\ell-k_{i}(\ell)}^{\ell-1} \frac{1}{\xi(j)+1}\right)
$$

(b) There exists a real solution sequence $\{\omega(n)\}$ of equation (10) which satisfies the inequality $\beta(n) \leq \omega(n) \leq \gamma(n)$ for $n \in \mathbf{N}_{M}$.

Proof (a) $\Rightarrow$ (b): We have to show that equation (10) has a solution sequence $\{\omega(n)\}$ for $n \in \mathbf{N}_{M}$. To this end, using Theorem $\mathrm{D}$, we prove that operator $S$, defined by (15), has a fixed point $\{\omega(n)\}$, which is a solution sequence of equation (10), and obviously satisfies the estimate $\beta(n) \leq \omega(n) \leq \gamma(n)$ for $n \in \mathbf{N}_{M}$.

Let now $M_{1}$ and $M_{2}$ be natural numbers such that $M \leq M_{1}<M_{2}<\infty$. Then the set

$$
\mathbf{N}_{1,2}=\left\{n \mid n \in \mathbf{N}, M_{1} \leq n \leq M_{2}\right\}
$$

is an arbitrary compact subset of the set $\mathbf{N}_{M}$. Set

$$
\begin{aligned}
& L:=\max _{M \leq n \leq M_{2}} \sum_{i=1}^{m}\left|P_{i}(n)\right|, \quad G:=\sup _{n \geq M} \gamma(n), \\
& B:=\min _{M \leq n \leq M_{2}} \beta(n), \quad K:=\max _{i=1}^{m} \max _{M \leq n \leq M_{2}} k_{i}(n), \\
& K_{B}:= \begin{cases}\max _{i=1}^{m} \max _{M \leq n \leq M_{2}} k_{i}(n), & -1<B \leq 0, \\
\min _{i=1}^{m} \min _{M \leq n \leq M_{2}} k_{i}(n), & B>0,\end{cases} \\
& K_{G}:= \begin{cases}\max _{i=1}^{m} \max _{M \leq n \leq M_{2}} k_{i}(n), & G>1, \\
\min _{i=1}^{m} \min _{M \leq n \leq M_{2}} k_{i}(n), & G<1 .\end{cases}
\end{aligned}
$$

Let

$$
F:=\left\{\{\xi(n)\} \in \mathbf{R}^{\infty}:|\xi(n)| \leq \mu(n), n \in \mathbf{N}_{M}\right\}, \quad \text { where } \mu(n)=\max _{n \geq n_{0}}\{|\gamma(n)|, 1\}
$$

It follows from assumptions (13) and (14) that the operator $S$, defined for $\{\xi(n)\} \in F$, satisfies the inequality $\sum_{\ell=n}^{\infty} \xi(\ell+1) \xi(\ell)<\infty$ and maps $F$ to $F$. It follows immediately from assumption (14) that the sequences in the image set $S F$ are uniformly bounded on any subset of $\mathbf{N}_{M}$. 
Let the sequence of sequences $\left\{\xi_{p}(n)\right\} \in F$ tend to the sequence $\{\xi(n)\}, p \rightarrow \infty$, uniformly on any finite interval of $N_{M}$, which means this convergence is uniform for all $n \in \mathbf{N}_{1,2}$.

In virtue of the following transformations:

$$
\begin{aligned}
& \left|\xi(\ell+1) \xi(\ell)-\xi_{p}(\ell+1) \xi_{p}(\ell)\right| \\
& \quad=\left|\xi(\ell+1) \xi(\ell)-\xi_{p}(\ell+1) \xi(\ell)+\xi_{p}(\ell+1) \xi(\ell)-\xi_{p}(\ell+1) \xi_{p}(\ell)\right| \\
& \quad \leq\left|\xi(\ell+1)-\xi_{p}(\ell+1)\right||\xi(\ell)|+\left|\xi(\ell)-\xi_{p}(\ell)\right|\left|\xi_{p}(\ell+1)\right|,
\end{aligned}
$$

we obtain that

$$
\sum_{\ell=n}^{M_{2}}\left|\xi(\ell+1) \xi(\ell)-\xi_{p}(\ell+1) \xi_{p}(\ell)\right| \leq 2 G \sum_{\ell=n}^{M_{2}+1}\left|\xi(\ell)-\xi_{p}(\ell)\right|
$$

and also we obtain that the inequalities

$$
\begin{aligned}
\prod_{j=\ell-k_{i}(\ell)}^{\ell-1} \frac{1}{\xi(j)+1}-\prod_{j=\ell-k_{i}(\ell)}^{\ell-1} \frac{1}{\xi_{p}(j)+1} & =\frac{\prod_{j=\ell-k_{i}(\ell)}^{\ell-1}\left(\xi_{p}(j)+1\right)-\prod_{j=\ell-k_{i}(\ell)}^{\ell-1}(\xi(j)+1)}{\prod_{j=\ell-k_{i}(\ell)}^{\ell-1}(\xi(j)+1)\left(\xi_{p}(j)+1\right)} \\
& \leq \frac{\left.(K-1) G^{K_{G}-1}+1\right) \sum_{j=\ell-k_{i}(\ell)}^{\ell-1}\left|\xi(\ell)-\xi_{p}(\ell)\right|}{\prod_{j=\ell-k_{i}(\ell)}^{\ell-1}(\beta(j)+1)\left(\beta^{p}(j)+1\right)} \\
& \leq \frac{\left(K G^{K G}+1\right) \sum_{j=\ell-K}^{\ell-1}\left|\xi(\ell)-\xi_{p}(\ell)\right|}{(B+1)^{2 K_{B}}}
\end{aligned}
$$

are valid. Using the previous inequalities, we can get the following transformations:

$$
\begin{aligned}
& \left|S \xi(n)-S \xi_{p}(n)\right| \\
& \leq \lim _{M_{2} \rightarrow \infty}\left(\sum_{\ell=n}^{M_{2}}\left|\xi(\ell+1) \xi(\ell)-\xi_{p}(\ell+1) \xi_{p}(\ell)\right|\right. \\
& \left.\quad+\sum_{\ell=n}^{M_{2}} \sum_{i=1}^{m}\left|P_{i}(\ell)\right|\left(\prod_{j=\ell-k_{i}(\ell)}^{\ell-1} \frac{1}{\xi(j)+1}-\prod_{j=\ell-k_{i}(\ell)}^{\ell-1} \frac{1}{\xi_{p}(j)+1}\right)\right) \\
& \leq \lim _{M_{2} \rightarrow \infty}\left(2 G \sum_{\ell=n}^{M_{2}+1}\left|\xi(\ell)-\xi_{p}(\ell)\right|+\frac{L\left(K G^{K_{G}}+1\right)}{(B+1)^{2 K_{B}}} \sum_{\ell=n}^{M_{2}} \sum_{j=\ell-K}^{\ell-1}\left|\xi(\ell)-\xi_{p}(\ell)\right|\right) .
\end{aligned}
$$

The uniform convergence of the sequence $\xi_{p}(n) \rightarrow \xi(n), p \rightarrow \infty$, for all $n \in \mathbf{N}_{1,2}$ implies that

$$
\left|\xi(n)-\xi_{p}(n)\right|<\delta \quad \text { for sufficiently large } p \text { and } n \in \mathbf{N}_{1,2} \text {. }
$$

If we use the following form of $\delta$ :

$$
\delta=\frac{\varepsilon}{C\left(M_{2}+2\right)}, \quad \text { where } C=2 G+\frac{L K\left(K G^{K}+1\right)}{(B+1)^{2 K_{B}}}
$$


we obtain

$$
\begin{aligned}
\left|S \xi(n)-S \xi_{p}(n)\right| & \leq \lim _{M_{2} \rightarrow \infty}\left(2 G \delta\left(M_{2}+2-n\right)+\frac{L\left(K G^{K_{G}}+1\right)}{(B+1)^{2 K_{B}}} K \delta\left(M_{2}+1-n\right)\right) \\
& <\lim _{M_{2} \rightarrow \infty}\left(2 G+\frac{L\left(K G^{K_{G}}+1\right)}{(B+1)^{2 K_{B}}} K\right) \delta\left(M_{2}+2\right) \\
& =\lim _{M_{2} \rightarrow \infty} C \frac{\varepsilon}{C\left(M_{2}+2\right)}\left(M_{2}+2\right)=\lim _{M_{2} \rightarrow \infty} \varepsilon=\varepsilon
\end{aligned}
$$

for $n \in \mathbf{N}_{1,2}$, if $p$ is sufficiently large. Thus, $S \xi_{p}(n) \rightarrow S \xi(n), p \rightarrow \infty$, uniformly on any finite subset $N_{M}$.

We obtained that the conditions of the Schauder-Tychonoff theorem are satisfied, and hence the mapping $S$ has at least one fixed point $\{\omega(n)\}$ in $F$. Moreover, because of the equality $\omega(n)=S \omega(n)$ for $n \in \mathbf{N}_{M}$, we conclude that $\{\omega(n)\}$ is the solution sequence of equation (10) with the property that $\beta(n) \leq \omega(n) \leq \gamma(n)$ for $n \in \mathbf{N}_{M}$. The first part of the proof is complete.

(b) $\Rightarrow$ (a): If $\{\omega(n)\}$ is a solution sequence of (10), then taking $\beta(n)=\gamma(n)=\omega(n)$ for $n \in \mathbf{N}_{M}$, the conditions of Theorem 1 are satisfied because of the fact that $\omega(n)=S \omega(n)$. The proof is complete.

\section{Existence of positive solutions}

Let the sequence $\{\mu(n)\}$ be such that $0<\mu(n)<1$, and set $\beta(n)=-\mu(n)$ and $\gamma(n)=\mu(n)$ in Theorem 1. Now we can formulate the conditions for the existence of positive solutions of equation (3).

Theorem 2 Assume that conditions $\left(\mathrm{H}_{1}\right)$ and $\left(\mathrm{H}_{2}\right)$ hold and there exists a positive sequence $\{\mu(n)\}$ for $n \in \mathbf{N}_{M}$ for some natural number $M \geq n_{0}$ such that $0<\mu(n)<1$ for $n \in \mathbf{N}_{M}$ and

$$
\sum_{\ell=n}^{\infty}\left(\mu(\ell+1) \mu(\ell)+\sum_{i=1}^{m}\left|P_{i}(\ell)\right| \prod_{j=\ell-k_{i}(\ell)}^{\ell-1} \frac{1}{1-\mu(j)}\right) \leq \mu(n)
$$

holds for $n$ large enough. Then equation (3) has a positive solution $\{a(n)\}$ for $n \in \mathbf{N}_{M}$.

Proof Let the sequence $\{\mu(n)\}$ be given such that the conditions of the theorem hold. Since $0<\mu(n)<1$, hence $-1<-\mu(n)<0$ and $0<1-\mu(n)<1$. We show that the conditions of Theorem 1 are satisfied with $\beta(n)=-\mu(n)$ and $\gamma(n)=\mu(n)$ for $n$ large enough.

Let $\{\xi(n)\}$ be a real sequence such that $|\xi(n)| \leq \mu(n)$. Because of the property that $-\mu(n) \leq \xi(n) \leq \mu(n)$, the inequality

$$
1-\mu(n) \leq \xi(n)+1 \quad \text { implies that } \quad \frac{1}{\xi(n)+1} \leq \frac{1}{1-\mu(n)} \quad \text { for } n \in \mathbf{N}_{M} .
$$

Because of assumption (16) and some transformations, it follows that

$$
\begin{aligned}
|S \xi(n)| & \leq \sum_{\ell=n}^{\infty}\left(|\xi(\ell+1)||\xi(\ell)|+\sum_{i=1}^{m}\left|P_{i}(\ell)\right| \prod_{j=\ell-k_{i}(\ell)}^{\ell-1} \frac{1}{\xi(j)+1}\right) \\
& \leq \sum_{\ell=n}^{\infty}\left(\mu_{\ell+1} \mu(\ell)+\sum_{i=1}^{m}\left|P_{i}(\ell)\right| \prod_{j=\ell-k_{i}(\ell)}^{\ell-1} \frac{1}{1-\mu(j)}\right) \leq \mu(n) .
\end{aligned}
$$


Therefore, in virtue of Theorem 1, equation (3) has a positive solution and the proof is complete.

Remark 1 The result of Theorem 2 is the discrete analogue of the result presented in Theorem A and generalizes the result given in [39] for first-order linear difference equations with variable delays.

Now we would like to find the sequence $\{\mu(n)\}$ in the form $\mu(n)=\frac{A}{n}$, where $A$ is some constant. Since

$$
\begin{aligned}
\sum_{\ell=n}^{\infty} \mu(\ell+1) \mu(\ell) & =\sum_{\ell=n}^{\infty} \frac{A^{2}}{\ell(\ell+1)}=\lim _{M \rightarrow \infty} \sum_{\ell=n}^{M} \frac{A^{2}}{\ell(\ell+1)}=\lim _{M \rightarrow \infty} \sum_{\ell=n}^{M}\left(\frac{A^{2}}{\ell}-\frac{A^{2}}{\ell+1}\right) \\
& =\lim _{M \rightarrow \infty}\left(\frac{A^{2}}{n}-\frac{A^{2}}{n+1}+\frac{A^{2}}{n+1}-\frac{A^{2}}{n+2}+\cdots+\frac{A^{2}}{M}-\frac{A^{2}}{M+1}\right)=\frac{A^{2}}{n}
\end{aligned}
$$

and

$$
\prod_{j=\ell-k_{i}(\ell)}^{\ell-1} \frac{1}{1-\mu(j)}=\prod_{j=\ell-k_{i}(\ell)}^{\ell-1} \frac{1}{1-\frac{A}{j}}=\prod_{j=\ell-k_{i}(\ell)}^{\ell-1} \frac{j}{j-A}
$$

condition (16) takes the form

$$
\sum_{\ell=n}^{\infty}\left(\sum_{i=1}^{m}\left|P_{i}(\ell)\right| \prod_{j=\ell-k_{i}(\ell)}^{\ell-1} \frac{j}{j-A}\right) \leq \frac{A}{n}-\frac{A^{2}}{n} .
$$

Choosing $A$ such that the function $f(A)=A-A^{2}$ takes the maximum value, we obtain $A=\frac{1}{2}$ and we can formulate the following corollary of Theorem 2 .

Corollary 1 Assume that conditions $\left(\mathrm{H}_{1}\right)$ and $\left(\mathrm{H}_{2}\right)$ hold and

$$
n \sum_{\ell=n}^{\infty}\left(\sum_{i=1}^{m}\left|P_{i}(\ell)\right| \prod_{j=\ell-k_{i}(\ell)}^{\ell-1} \frac{2 j}{2 j-1}\right) \leq \frac{1}{4}
$$

holds for $n$ large enough. Then equation (3) has an eventually positive solution.

In particular, if the sequences of delays $\left\{k_{i}(n)\right\}(i=1,2, \ldots, m)$ are bounded by $K$, we have

$$
\sum_{\ell=n}^{\infty}\left(\sum_{i=1}^{m}\left|P_{i}(\ell)\right| \prod_{j=\ell-k_{i}(\ell)}^{\ell-1} \frac{2 j}{2 j-1}\right) \leq\left(\frac{2(n-K)}{2(n-K)-1}\right)^{K} \sum_{\ell=n}^{\infty}\left(\sum_{i=1}^{m}\left|P_{i}(\ell)\right|\right)
$$

and

$$
\limsup _{n \rightarrow \infty}\left(\frac{2(n-K)}{2(n-K)-1}\right)^{K}=1
$$

Hence, we obtain the following non-oscillation criterion. 
Corollary 2 Assume that conditions $\left(\mathrm{H}_{1}\right)$ and $\left(\mathrm{H}_{2}\right)$ hold and the sequences of delays $\left\{k_{i}(n)\right\}$ $(i=1,2, \ldots, m)$ are bounded. If

$$
\limsup _{n \rightarrow \infty} n \sum_{\ell=n}^{\infty} \sum_{i=1}^{m}\left|P_{i}(\ell)\right| \leq \frac{1}{4}
$$

holds, then equation (3) has an eventually positive solution.

Remark 2 The result of Corollary 2 is the discrete analogue of the result presented in Theorem $\mathrm{B}$ and at the same time generalizes the result given in Theorem $\mathrm{C}$ for secondorder linear difference equations with variable delays.

\section{Competing interests}

The author declares that she has no competing interests.

\section{Authors' contributions}

Since there is one author, she completed all tasks necessary for the article

\section{Acknowledgements}

The research is supported by the Serbian Ministry of Science, Technology and Development for Scientific Research Grant no. III44006. The author thanks the referees for the valuable comments.

Received: 18 September 2012 Accepted: 8 March 2013 Published: 29 March 2013

\section{References}

1. Diblik, J, Ružičková, M, Šmarda, Z, Šuta, Z: Asymptotic convergence of the solutions of a dynamic equation on discrete time scales. Abstr. Appl. Anal. 2012, Article ID 580750 (2012)

2. El-Sheikh, MMA, Abd Alla, MH, El-Maghrabi, EM: Oscillation and nonoscillation of nonlinear second order difference equations. J. Appl. Math. Comput. 21(1-2), 203-214 (2006)

3. He, XZ: Oscillatory and asymptotic behaviour of second order nonlinear difference equations. J. Math. Anal. Appl. $175,482-498(1993)$

4. Huiqin, C, Zhen, J: Oscillation criteria of solution for a second order difference equations with forced term. Discrete Dyn. Nat. Soc. 2000, Article ID 171234 (2000)

5. Koplatadze, R, Kvinikadze, G: Necessary conditions for the existence of positive solutions of second-order linear difference equations and sufficient conditions for the oscillation of solutions. Nonlinear Oscil. 12(2), 184-198 (2009)

6. Krasznai, B, Gyori, I, Pituk, M: Positive decreasing solutions of higher-order nonlinear difference equations. Adv. Differ. Equ. 2010, Article ID 973432 (2010)

7. Li, WT, Fan, XL, Zhoung, C: On unbounded positive solutions of second-order difference equations with a singular nonlinear term. J. Math. Anal. Appl. 246, 80-88 (2000)

8. Liu, B, Cheng, SS: Nonoscillatory solutions of a second-order difference equation of Poincaré type. J. Math. Anal. Appl. 204, 482-493 (1996)

9. Medina, R, Pituk, M: Positive solutions of second order nonlinear difference equations. Appl. Math. Lett. 22, 679-683 (2009)

10. Tang, $\mathrm{XH}$ : Bounded oscillation of second order delay difference equations of unstable type. Comput. Math. Appl. 44, 1147-1156 (2002)

11. Tang, $\mathrm{XH}, \mathrm{Yu}$, JS: Oscillation of delay difference equations in a critical state. Appl. Math. Lett. 13, 9-15 (2000)

12. Zhang, BG: Oscillation and asymptotic behavior for second-order difference equations. J. Math. Anal. Appl. 173, 58-68 (1993)

13. Zhang, Z, Li, Q: Oscillation theorems for second-order advanced functional difference equations. Comput. Math. Appl. 36(6), 11-18 (1998)

14. Baštinec, J, Diblik, J, Šmarda, Z: Existence of positive solutions of discrete linear equations with a single delay. J. Differ. Equ. Appl. 16(9), 1047-1056 (2010)

15. Baštinec, J, Diblik, J, Šmarda, Z: An explicit criterion for the existence of positive solutions of the linear delayed equation $\dot{x}(t)=-c(t) x(t-\tau(t))$. Abstr. Appl. Anal. 2011, Article ID 561902 (2011)

16. Baštinec, J, Diblik, J: One case of appearance of positive solutions of delayed discrete equations. Appl. Math. 48(6), 429-436 (2003)

17. Baštinec, J, Diblik, J: Subdominant positive solutions of the discrete equation $\Delta u(k+n)=-p(k) u(k)$. Abstr. Appl. Anal. 6, 461-470 (2004)

18. Čermak, J: On the related asymptotics of delay differential and difference equations. Dyn. Syst. Appl. 14(3-4), 419-430 (2005)

19. Čermak, J: Asymptotic bounds for linear difference systems. Adv. Differ. Equ. 2010, Article ID 182696 (2010)

20. Deng, J: A note of oscillation of second-order nonlinear difference equation with continuous variable. J. Math. Anal. Appl. 280, 188-194 (2003) 
21. Došly, O, Fišnarová, S: Linearized Riccati technique and (non-)oscillation criteria for half-linear difference equations. Adv. Differ. Equ. 2008, Article ID 438130 (2008)

22. Hille, E: Non-oscillation theorems. Trans. Am. Math. Soc. 64, 234-252 (1948)

23. Jaroš, J, Stavroulakis, IP: Oscillation tests for delay equations. Rocky Mt. J. Math. 29(1), 197-207 (1999)

24. Thandapani, E, Ravi, K, Graef, JR: Oscillation and comparison theorems for half-linear second-order difference equations. Comput. Math. Appl. 42, 953-960 (2001)

25. Yang, X: Nonoscillation criteria for second-order nonlinear differential equations. Appl. Math. Comput. 131, 125-131 (2002)

26. Agarwal, RP: Difference Equations and Inequalities, Theory, Methods and Applications. Dekker, New York (2000)

27. Agarwal, RP, Bohner, M, Grace, SR, O'Regan, D: Discrete Oscillation Theory. Hindawi Publishing Corporation, New York (2005)

28. Kusano, T, Naito, Y, Ogata, A: Strong oscillation and nonoscillation of quasilinear differential equations of second order. Differ. Equ. Dyn. Syst. 1(2), 1-10 (1994)

29. Kusano, T, Yoshida, N: Nonoscillation theorems for a class of quasilinear differential equations of second order J. Math. Anal. Appl. 189, 115-127 (1995)

30. Péics, H, Karsai, J: Existence of positive solutions of halflinear delay differential equation. J. Math. Anal. Appl. 323, 1201-1212 (2006)

31. Baštinec, J, Berezansky, L, Diblik, J, Šmarda, Z: A final result on the oscillation of solutions of the linear discrete delayed equation $\Delta x(n)=-p(n) x(n-k)$ with a positive coefficient. Abstr. Appl. Anal. 2011, Article ID $586328(2011)$

32. Berezansky, L, Diblik, J, Šmarda, Z: Positive solutions of second-order delay differential equations with a damping term. Comput. Math. Appl. 60(5), 1332-1342 (2010)

33. Lei, C: Remarks on oscillation of second-order linear difference equation. Appl. Math. Comput. 215, $2855-2857$ (2009)

34. Li, X, Jiang, J: Oscillation of second-order linear difference equations. Math. Comput. Model. 35, $983-990$ (2002)

35. Zhou, Y, Zhang, BG: Oscillation of delay difference equations in a critical state. Comput. Math. Appl. 39, 71-80 (2000)

36. Brooks, RM, Schmitt, K: The contraction mapping principle and some applications. In: Monograph 09, San Marcos: Electron. J. Differential Equations, Department of Mathematics, Texas State University - San Marco 2009:90

37. Coppel, WA: Stability and Asymptotic Behavior of Differential Equations. Hindawi Publishing Corporation, Heath (1965)

38. Isträtescu, VI: Fixed Point Theory. An Introduction. Reidel, Dordrecht (1981)

39. Péics, H: Applications of generalized characteristic equation of linear delay difference equations. Mat. Vesn. 50, 31-36 (1998)

doi:10.1186/1687-1847-2013-82

Cite this article as: Peics: Positive solutions of second-order linear difference equation with variable delays. Advances in Difference Equations 2013 2013:82

\section{Submit your manuscript to a SpringerOpen ${ }^{\circ}$ journal and benefit from:}

- Convenient online submission

- Rigorous peer review

- Immediate publication on acceptance

- Open access: articles freely available online

- High visibility within the field

- Retaining the copyright to your article 